\title{
Diffusion tubes: a method for the mass culture of ctenophores and other pelagic marine invertebrates
}

\author{
Wyatt L Patry ${ }^{\text {Corresp., } 1}{ }^{\text {, MacKenzie Bubel }}{ }^{1}$, Cypress Hansen ${ }^{1}$, Thomas Knowles ${ }^{1}$ \\ ${ }^{1}$ Animal Care Division, Monterey Bay Aquarium, Monterey, California, United States of America \\ Corresponding Author: Wyatt L Patry \\ Email address: wpatry@mbayaq.org
}

The culture of pelagic marine invertebrates, especially the ctenophore Mnemiopsis leidyi, has been demonstrated in past studies dating back to the 1960's, however the mass culture of delicate pelagic invertebrates has remained elusive. By using a pair of acrylic tubes and enabling water diffusion between them, we have been able to reliably and cost effectively mass culture several genera of ctenophores (Pleurobrachia, Hormiphora, Bolinopsis, Mnemiopsis, and Leucothea), one species of siphonophore (Nanomia) and one species of larvacean (Oikopleura). The simple, compact method is effective enough to support two permanent exhibits of ctenophores at the Monterey Bay Aquarium while minimizing live food culture requirements with the potential to support further investigation of pelagic marine invertebrate ontogeny, ecology and genomics. 


\section{Diffusion tubes: a method for the mass culture of ctenophores}

\section{3 and other pelagic marine invertebrates.}

5 Wyatt L. Patry ${ }^{1 *}$, MacKenzie Bubel ${ }^{1}$, Cypress Hansen ${ }^{1}$, Thomas Knowles ${ }^{1}$

$7{ }^{1}$ Animal Care Division, Monterey Bay Aquarium, Monterey, California, United States of

8 America

$10 *$ Corresponding author

11 E-mail: wpatry@mbayaq.org

13 Abstract

15 The culture of pelagic marine invertebrates, especially the ctenophore Mnemiopsis leidyi, has

16 been demonstrated in past studies dating back to the 1960's, however the mass culture of delicate

17 pelagic invertebrates has remained elusive. By using a pair of acrylic tubes and enabling water

18 diffusion between them, we have been able to reliably and cost effectively mass culture several

19 genera of ctenophores (Pleurobrachia, Hormiphora, Bolinopsis, Mnemiopsis, and Leucothea),

20 one species of siphonophore (Nanomia) and one species of larvacean (Oikopleura). The simple,

21 compact method is effective enough to support two permanent exhibits of ctenophores at the 
22 Monterey Bay Aquarium while minimizing live food culture requirements with the potential to

23 support further investigation of pelagic marine invertebrate ontogeny, ecology and genomics.

25 Introduction

27 Interest in ctenophore culture and other pelagic marine invertebrates as model organisms has 28 surged recently (Howes et al., 2014; Jaspers et al., 2015, Jaspers et al., 2018, Martí-Solans et al., 29 2015; Presnell and Browne, 2019). Basic ontogeny of ctenophores has been observed since at 30 least the turn of the 19th century by Chun, Agassiz and Mayer however maintaining cultures in

31 the laboratory remained elusive until later in the $20^{\text {th }}$ century. Pioneering efforts in developing

32 rearing vessels for pelagic culture were made by Wulf Greve with his invention of the

33 planktonkreisel (Greve 1968 and 1970) and other variations such as the double cuvette design

34 (Greve, 1975). Greve achieved several generations of Pleurobrachia pileus in these studies. The

35 kreisel was later formalized by Hamner (1990) for use aboard ships and then adapted for use in

36 public aquariums by the Monterey Bay Aquarium (Raskoff et al., 2003).

The method presented here originated at the Monterey Bay Aquarium in July 2015 to support the temporary exhibition, The Jellies Experience, and while collaborating with W. E. Browne of University of Miami on Mnemiopsis leidyi culture (Presnell et al. 2019). After successfully

41 culturing Mnemiopsis, we turned our focus to culturing other ctenophores such as Pleurobrachia

42 bachei and Bolinopsis infundibulum. The young cydippids of these species are so delicate that

43 they do not survive in traditional plankton kreisels. They are susceptible to being damaged by the 44 seawater flow in the tank and sticking to the outflow screen. Additionally, juveniles do not 
45 survive in standing dishes of seawater due to rapid accumulation of ammonia and high mortality

46 from being transferred into new seawater. Therefore, we needed a rearing tank with minimal or

47 no mechanical driven water flow, no outward pressure on the outflow screen, and the ability to

48 passively exchange new seawater. We experimented with rearing tanks featuring seawater flow

49 just outside of the outflow screen, such that seawater could passively exchange without creating

50 outward pressure on the screen. While these methods were effective at eliminating buildup of

51 nitrogenous wastes and minimizing forces on juvenile ctenophores caused by seawater flow, the

52 tank shapes were not appropriate for the swimming and feeding behavior of the animals. In

53 traditional rectangular aquaria, juveniles were observed actively consuming prey at or near the

54 surface and then sinking to the bottom, where significant biofouling occurs. Initially we designed

55 and constructed a cylindrical tank with more vertical space for feeding, minimal biofouling

56 surface area with mesh covering the bottom and secured in a pseudo-kreisel such that water

57 flowed up through the bottom. This initial design proved somewhat effective, however juveniles

58 still spent significant time contacting the bottom mesh. An improved design providing even more

59 vertical space, while reducing the volume of the tank was implemented. Our final version utilizes

60 a double cylinder configuration in which an elongated inner cylinder is surrounded by an outer

61 cylinder 'sleeve', affectionately referred to as a "cteno-tube" in the lab.

62

63 Over the past four years, we have experimented with this new double cylinder rearing tank

64 design (Fig. 1) and have refined a protocol for raising fragile pelagic ctenophores. We also

65 performed a single experiment using several flow rate treatments to observe a number of

66 physical parameters ( $\mathrm{pH}$, oxygen and temperature) within the 'cteno-tube' in relation to adult

67 ctenophore yield. Here we present these results, additional variations on the double-cylinder 
68 method and provide a best practices protocol for the mass culture of pelagic ctenophores and

69 other gelata.

71 Method

Materials

Diffusion tubes were constructed using two $0.9 \mathrm{~m}$ long acrylic tubes. The outer and inner tubes had volumes of $\sim 35 \mathrm{~L}$ and $28 \mathrm{~L}$ with inner diameters of $21.59 \mathrm{~cm}$ and $20 \mathrm{~cm}$ respectively, so that one could be placed inside the other (an inner and outer tube, Fig. 2a, b). Each acrylic tube had a thickness of $0.3175 \mathrm{~cm}$. The bottom of the inner tube was fitted with $55 \mu \mathrm{m}$ nylon screening (Model\# M55 PentairAES, Inc., Fig. 2e) using silicone sealant (Dowsil 795 or 999-A, Fig 2j). This inner tube was then placed on a $3 \mathrm{~cm}$ tall riser made of rigid plastic mesh (Model\# N1020 PentairAES, Inc., Fig. 2g) such that the top of the inner tube rises above the outer cylinder (Fig.

82 Step. 7). The outer cylinder was glued to a $\sim 1 \mathrm{~cm}$ thick square acrylic baseplate (Fig. 2c), 15 -

$8320 \mathrm{~cm}^{2}$, using acrylic cement (Weld-On \#16 Fast Set solvent cement, Fig. 2d). Rigid PVC tubing,

$840.635 \mathrm{~cm}$ inner diameter, (Part\# 48855K41, McMaster-Carr) was placed in the outer tube,

85 between tubes, providing water flow near the bottom. Filtered seawater $(5 \mu \mathrm{m})$ was pumped

86 through the tubing at rates of 1.1, 2.2 and 4.5 liters per minute (Lpm). Three identical pairs of

87 diffusion tubes were constructed and placed on a wet table with recirculating filtered seawater (5

$88 \mu \mathrm{m})$ such that water overflowed out of the outer cylinder and on to the table. Diffusion between

89 tubes through the $55 \mu$ m nylon screening was observed by the addition of dye (McCormick blue

90 food coloring) to the incoming water. In the $4.5 \mathrm{Lpm}$ treatment, flow was such that a slight 
91 suction vortex pulled the ctenophore eggs into the bottom screen which prevented hatching. To

92 resolve this, a PVC pipe tee fitting (Part\# 4881K47, McMaster-Carr) was added to the bottom of

93 the inflow tube to reduce water velocity and divert the incoming water away from directly

94 underneath the screen, which proved successful. All current materials and pricing are listed on

95 the "CtenoTube" repository Wiki on GitHub (https://github.com/wyattp11/CtenoTube/wiki) as

96 of this writing the cost for a single diffusion tube setup is $\sim \$ 465$ including all tools necessary for 97 assembly.

Assembly Instructions

101 Outer tube construction (Fig. 2B): center the tube with the widest internal diameter (a) on the 102 base plate (c). Use the syringe (i) to draw up some acrylic glue into the syringe. Inject acrylic 103 glue into the seam between (a) and (c). Leave the tube and base plate to cure for at least 24 hours 104 before handling again. Cut screen (Fig. 2C): cut a circular piece from the sheet of screening (e) 105 using scissors (h). The diameter should be at least $2 \mathrm{~cm}+$ the diameter of the inner tube (b) to 106 allow enough overlap to glue down. Glue inner tube (Fig. 2D): Place a bead of silicone sealant

107 (j) around the top edge of the inner tube (b) and then about $1 \mathrm{~cm}$ below the edge (red lines).

108 Smear the lower bead so that it is smooth and flat using a gloved finger, ice or other flat edge.

109 Secure mesh on inner tube (Fig. 2E): Use the rubber band (f) to secure the piece of cut screen

110 from Step 2 over the end of inner tube with the silicone sealant (j). Place another bead of silicone

111 over the mesh and smooth until the screening is no longer visible. The edge of the tube can also

112 receive a light bead and smoothing. Construct riser (Fig. 2F): Cut a $3 \mathrm{~cm}$ tall length of the rigid

113 plastic mesh (g) that matches the circumference of the inner tube (b). Remove any sharp edges, 
114 edges should be smooth. Use a pair of small zip ties (k) to secure the riser to itself, forming a

115 tube. To avoid the riser puncturing the fine screen (e) a piece of cut rubber tubing may be placed

116 on the top edge of the riser. Place the finished riser at the bottom of the outer tube (Fig. 2G).

117 Now place the inner tube (b) inside the outer tube (a) so it gently rests on the riser (Fig. 2F). The

118 tube setup is now ready for filtered seawater. The supply line may be rigid acrylic tubing or

119 flexible PVC tubing.

120

121

122 Characterizing physical parameters

123

124 A set of probes (Hach HQ40d with LDO probe) for measuring $\mathrm{pH}$, dissolved oxygen, and 125 temperature was deployed within the inner cylinder of each set of tubes and reads were taken 126 every $12.7 \mathrm{~cm}$ at seven discrete depths. Tubes were allowed to equilibrate for at least 24 hours

127 prior to testing and the probes were lowered slowly to read depths using a simple pulley system.

128 A minimum settling time of two minutes at each depth was necessary to take accurate reads of

129 each parameter. We also used a dye to observe mixing/stratification within the tube and found

130 that the inflow tube caused flow disturbance resulting in water primarily diffusing in from the

131 opposite side of the tube inflow. In order to account for discrepancies caused by the inflow

132 tube's position on one side vs. the other, probe readings were established twice, once closest to

133 the inflow tube and once on the opposite side.

134

135 Methods of spawning and culturing

136 
137 Three adult Hormiphora californensis were placed in each inner cylinder (Fig. 3a). To induce

138 spawning (Fig. 3b), the tubes were shrouded in a black, opaque cover for total darkness

139 overnight, followed by 2 hours of bright light the next morning (Kessil A360 Tuna Blue LED

140 aquarium light placed $\sim 5 \mathrm{~cm}$ above the inner cylinder) (Pang \& Martindale 2008). When eggs

141 were observed throughout the water column, usually $\sim 12$ hours post spawn or 24 hours after

142 setup, the adults were removed from the tubes using a spoon attached to a short pole or by slowly

143 swirling them to the surface.

144 Hatching of the eggs was observed 12 - 48 hours post spawning. Juvenile cydippids were fed on

145 the first day post hatch (dph). Each tube was given a standardized concentration ( $\sim 30$ nauplii $\mathrm{mL}^{-}$

$\left.14{ }^{1}{ }^{1}\right)$ of live Parvocalanus crassirostris (Reed Mariculture Inc.) nauplii mixed with live algae

147 (Isochrysis galbana, Rhodomonas lens, Dunaliella tertiolecta, or Tetraselmis chuii, Florida Aqua

148 Farms, Inc.) in a 1:1 ratio, both cultured in the lab. P. crassirostris nauplii were added at the

149 surface of the tube water column for six weeks using the following regimen: $25 \mathrm{~mL} 3$ times per

150 week (Sunday, Tuesday, Thursday) for the first 14 days, increasing to $50 \mathrm{~mL}$ for the next 14

151 days, and $100 \mathrm{~mL}$ for the last 14 days.

152 The ctenophores were observed throughout the water column and sampled once a week for six

153 weeks to be photographed and measured. For photo documentation and measurement, 15 - 30

154 juvenile cydippids were randomly pipetted out of the water column down to $30 \mathrm{~cm}$ depth and

155 transferred to a glass crystallizing dish filled with filtered seawater for photographing under a

156 microscope (Zeiss Stereo Zoom V16, Canon EOS Rebel T5i). Each photograph was scaled and

157 measured using ImageJ 1.52A (Schneider et al., 2012).

158

159 Post-diffusion tube rearing 
161 When cydippids reached $\sim 0.5-1 \mathrm{~cm}$ in diameter $(\sim 30 \mathrm{dph})$ they were transferred using widened

162 disposable polyethylene pipettes (tips cut off, Transfer pipets 13-711-7M, FisherBrand) or small

163 polypropylene beakers (50 - $100 \mathrm{~mL}$ Nalgene Griffin low form beaker) from the diffusion tubes

164 to kreisel tanks (Raskoff et al., 2003) modified to provide for better longevity of ctenophores.

165 The kreisel screens were replaced with a polyethylene mesh with 4 mm openings (Model

166 \#N1670 PentairAES, Inc.). Slot openings in the supply boxes were reduced in width using a

167 single piece of $4 \mathrm{~mm}$ corrugated plastic (high-density polyethylene and polypropylene, Model

$168 \#$ 24244SC CorrugatedPlastics.Net) instead of two $6 \mathrm{~mm}$ pieces which are typically found as

169 filler for $12.7 \mathrm{~mm}$ slot openings. Pumps were set to provide $\sim 10$ liters per minute (Lpm) of

170 volumetric flow to the lower supply box and 7.5 Lpm to the upper box (Model DCW-2000

171 Jebao, Inc.) using flow meters (Blue-White Industries, Ltd, Model \#F-44500L-8). The upper box

172 provided $\sim 0.8 \mathrm{Lpm}$ of filtered seawater $(5 \mu \mathrm{m})$ at $13^{\circ} \mathrm{C}$. The diet was diversified to include $1-2$

173 mysid shrimp (Mysidopsis bahia, Aquatic Indicators, Inc.) per comb jelly, usually 200 per

174 kreisel every 2 days and $200 \mathrm{~mL}$ of $P$. crassirostris adult copepods fed daily around noon.

176 Non-ctenophore rearing

177

178 Oikopleura sp. and Nanomia bijuga were reared in much the same manner as the ctenophores.

179 Up to 10 adults of Oikopleura were used to start a culture in a diffusion tube setup, however this 180 is not typically feasible with Nanomia due to the length of the wild colonies which will tangle

181 when more than 3-4 adults are added to an inner tube of $15 \mathrm{~cm}$ diameter. If adult Nanomia are to

182 be retained after spawning, then the number of adults should be limited based on the diameter of 
183 inner tube used. Culture temperature for Nanomia was manipulated, adults were spawned at

$18413^{\circ} \mathrm{C}$ and then the temperature raised to $18^{\circ} \mathrm{C}$ for the planulae to begin development. At colder

185 temperatures Nanomia had very low colony development $\left(14^{\circ} \mathrm{C}\right)$ or no development whatsoever

$186\left(7^{\circ} \mathrm{C}\right)$. Once multi-tentacle Nanomia reach $\sim 5 \mathrm{~cm}$ non-contracted length they can be acclimated

187 back down to $14^{\circ} \mathrm{C}$ and graduated to a pseudo-kreisel (traditional kreisels do not seem to work

188 well for adult Nanomia as the pneumatophores collide with curved acrylic surfaces too often,

189 while they are well adapted to touching the surface of the water in a pseudo-kreisel). Adult

190 Nanomia required live mysids (Mysidopsis bahia) while in the pseudo-kreisel to reach maturity,

191 which occurs rapidly over the course of a week at $14^{\circ} \mathrm{C}$. As of this writing, F1 Nanomia (ranging

192 in non-contracted lengths of $15-25 \mathrm{~cm}$ ) were spawned and successfully to F2 individuals using

193 the proposed protocol (see conclusions). Oikopleura culture was performed just once at $13^{\circ} \mathrm{C}$

194 and a new generation appeared every week with regular feedings of live algae however it was

195 unclear if 3 or 4 generations were produced due to the rapid succession. It is not necessary to

196 remove adult Oikopleura after spawning however it may be advantageous to remove discarded

197 mucous houses. At the end of $\sim 30$ days the screen had clogged with algae, perhaps mucous, and

198 the culture was lost. Neither Oikopleura or Nanomia were part of the experimental results

199 presented in this paper.

200

201 Statistical analysis

202

203 Mean length data was analyzed using R (R Core Team, 2017). Growth rates were adapted to the

204 data using the R package Growthcurver (https://cran.r-project.org/package=growthcurver).

205 Descriptive statistics, Kruskal-Wallis ANOVA and Wilcoxon pairwise comparisons were made 
206 using packages dplyr(https://CRAN.R-project.org/package=dplyr),

207 ggpubr(https://rpkgs.datanovia.com/ggpubr/) and ggplot2(http://ggplot2.tidyverse.org/).

208

209 Results

210

211 Physical parameters and depth profiling

212

213 Temperature

214

215 Under the highest turnover rate (4.5 Lpm), the water column exhibited a significant thermocline 216 within $12-24 \mathrm{~cm}$ of the surface. With a surface temperature between $16-17^{\circ} \mathrm{C}$, the surface layer

217 was approximately 3-4 degrees warmer than the water below $24 \mathrm{~cm}$ in depth (Fig. 4a). This was

218 in agreement with dye observations, which indicated slower turnover in the top half of the water

219 column under the $4.5 \mathrm{Lpm}$ treatment. Although the other two treatments exhibited less dramatic

220 thermoclines, the mean surface temperature exceeded the mean bottom temperature by at least

$2211.5^{\circ} \mathrm{C}$ in all three treatments. Temperatures reported are the mean of the measurements taken on

222 the two opposite sides of the inner cylinder, nearest and farthest from the inflow line. No

223 significant difference was found between the near and far side measurements.

224

$225 \mathrm{pH}$

226

$227 \mathrm{pH}$ was the most consistent of all parameters measured, ranging between 7.84 and 7.90 across all

228 flow rates and depths, with the low flow treatment yielding the lowest $\mathrm{pH}$ at the surface of the 
229 water column (Fig. 4b). The low and high flow treatments exhibited slightly decreased $\mathrm{pH}$ at the

230 surface layer.

231

232 Dissolved Oxygen

233

234 Dissolved oxygen measurements exhibited no significant differences with regard to depth, flow 235 rate, or temperature. The high flow treatment showed slightly decreased dissolved oxygen at the 236 surface layer in conjunction with decreased $\mathrm{pH}$, however the measurements were within the 237 standard error $( \pm 0.02 \mathrm{pH}, \pm 0.2 \mathrm{mg} / \mathrm{L} \mathrm{DO})$ of the probe (Fig. $4 \mathrm{c})$.

238

239 Culturing success

240

241 All three treatments were successful in raising $>50 \mathrm{H}$. californensis over a six-week period. The

242 high flow (4.5 L pm) treatment yielded the most cydippids (89), followed by medium flow (2.2

243 Lpm) (87) and low flow (1.1 Lpm) (75). The mean cydippid length of each treatment varied little

244 from 0-23 dph (each p > 0.07) and then greatly increased by $30 \mathrm{dph}$ (Fig. 5). Cydippids in the

245 high flow treatment had the highest mean lengths at the end of the experiment (Wilcoxon signed-

246 rank test, $\mathrm{p}=0.00046$ and 2.1 E-4, low vs. high and medium vs. high respectively), while the

247 medium treatment had the smallest mean lengths (Fig. 6). Growthcurver produced best fits and

248 growth rates for the low and high flow treatments but failed to find a best fit for the medium flow

249 treatment (Table 1).

250

251 Discussion 
253 Our method utilizes a cylindrical tank shape which maximizes the ratio of water volume to the 254 surface area of tank bottom, which has several important effects. By minimizing the bottom 255 biofouling area, contact with these fouled surfaces is greatly decreased, vertical swimming or 256 migration space for juveniles is maximized and a thermocline is produced where the juvenile 257 cydippids can feed furthest from the tank bottom. Another key feature of our design is the 258 passive exchange of seawater through the mesh which generates much lower water velocities 259 than in a traditional plankton kreisel. These lower flow rates allow delicate juvenile ctenophores 260 to develop in a very gentle environment while still facilitating the exchange of clean filtered 261 seawater. In contrast to traditional aquaria for gelatinous zooplankton, our method provides a 262 superior pelagic environment for young ctenophores and other gelata larvae that is inexpensive 263 and easily replicable. At the time of this writing a single diffusion tube setup costs $\sim \$ 300$ USD, a 264 small pseudo-kreisel $(<0.5 \mathrm{~m} \mathrm{D})$ can cost twice that of a diffusion tube setup and takes up 2-3 265 times as much surface area in the lab which makes replication challenging. Reliable, replicable 266 mass culture of ctenophores has also not been demonstrated in traditional kreisels yet.

268 Using the highest turnover rate generated a more pronounced vertical thermocline and resulted in 269 higher survival and significantly faster growth rates among the treatments. It is not clear why the 270 low flow outperformed the medium flow treatment in mean length at $35 \mathrm{dph}$. Flow rate was a 271 critical factor in our previous runs with the diffusion tubes, if the flow rate was too low, we 272 observed pre-mature die-off of the culture. So, it may be that the medium and low flows are 273 actually equal in performance and the higher flow rate the ideal. Should flows need to be 274 minimized in a future lab setting, perhaps replication will provide better resolution. Replication 
275 of this experiment should produce a clear quantitative answer to this specific problem. While the

276 method has been replicated many times, this specific experiment testing flow was only

277 performed once.

278

279 Overall, fewer $H$. californensis were produced in this experiment than previous and subsequent

280 trials with the diffusion tubes. An F4 population was achieved shortly after the experiment using

28110 parents of the $\mathrm{F} 3$ population, yielding $>600$ cydippids and a similar run with $10 \mathrm{~F} 4$ parents

282 produced $>500$ cydippids to create the F5 generation currently in culture (growth and flow data

283 were not collected for these runs). We believe this was primarily due to the restricted quantities

284 of food used for this experiment (in previous use of this method feeding amounts varied between

285 just $25 \mathrm{~mL}$ up to $200 \mathrm{~mL}$ of copepod nauplii, depending on the specific user). When feeding

286 quantity was increased from $50 \mathrm{~mL}$ to $100 \mathrm{~mL}$ at week 5 there was a notable increase in growth

287 rate across treatments at $40 \mathrm{dph}$. It is also possible that lower yields may reflect use of fewer

288 parents $(\mathrm{n}=3)$ than previous/subsequent trials $(\mathrm{n}=10)$ due to inbreeding depression from more

289 self-fertilization. Ultimately, we have adopted a feeding regime that doubled the amounts of

290 copepod nauplii and uses 10 adults to keep the yield higher (see Conclusions).

291

292 Further development of this method has the potential for increased efficiency and work with new 293 species of gelata. Changing cylinder height and width may allow for more variation in

294 thermocline profiles and vertical space, however bigger tubes will require more volumetric flow

295 to the outer cylinder. For larger ctenophore species diffusion tubes with larger diameters may

296 have increased utility (Table 2), for example Leucothea pulchra was successfully cultured to F2

297 using diffusion tubes $2 \mathrm{~m}$ high and $0.35 \mathrm{~m}$ diameter with a flow of $\sim 4 \mathrm{Lpm}$ (Fig. 7). Bolinopsis 
298 infundibulum and Nanomia bijuga were grown using an inner cylinder measuring $0.9 \mathrm{~m}$ high and

$2990.25 \mathrm{~m}$ diameter (outer cylinder was $0.9 \mathrm{~m}$ high and $0.30 \mathrm{~m}$ diameter) (Fig. 8). Changing the

300 mesh size may impact diffusion rates between the two tubes, thermocline formation and perhaps

301 be better fitted to a particular target species. For example, a $22 \mu \mathrm{m}$ mesh was originally used for

302 P. bachei but we found the mesh fouled quickly. When replaced with $55 \mu \mathrm{m}$ mesh, diffusion and

303 flow performance were enhanced and fouling no longer a problem.

305 Conclusions

307 After four years of trials culturing various gelata using different sizes of the diffusion tubes, we 308 derived a standard procedure for mass culture of ctenophores from 1-10 parental adults using 1

$309 \mathrm{~m}$ diffusion tubes (Hormiphora and Pleurobrachia) and a feeding regime based on results

310 yielding $>100$ adult ctenophores in about 30 days was adopted as follows:

311 I. To start a culture from wild collected adults, use $1-10$ adult ctenophores depending on

312 desired genetic diversity. Adults should be fed a fish or mysid meal 24 hours prior to

313 spawning and again 1 hour before the dark period begins (Presnell et al., 2019).

314 II. To induce spawning, place all adults in an equilibrated diffusion cylinder (for inner

315 diameter $20 \mathrm{~cm}$, a flow rate of $4.5 \mathrm{Lpm}$ ), shrouded in opaque material to ensure complete

316 darkness for a 4-hour period (or overnight). Uncover the tube and expose to bright

317 lighting for at least 2 hours (Presnell et al., 2019).

318 III. When adults have spawned and eggs are visible in the water column, gently remove the 319 adults from the tube using ladles or dishes. 
320 IV. Eggs hatch within 12-48 hours, first feeding should ideally take place within the first 24

321 hours of hatching to ensure that cydippids are able to begin feeding immediately.

322 V. Feeding schedule: see Fig. 9.

323 VI. At 30 dph the cydippids may be moved to a pseudo-kreisel or kreisel tank. If cydippids 324 have not achieved a total body size of $0.5-1 \mathrm{~cm}$ diameter by this time, their residence in the tube may be increased to as long as $40 \mathrm{dph}$. However uniformly slow growth rates are a sign of a weak hatch, not enough food and/or significant disruption in water flow/diffusion through the tube. As residence time increases in the tube, so will impaction of the screen.

330 Gelatinous zooplankton require physically stable conditions to grow en masse. Our tube design

331 achieves passive water exchange via diffusion, minimizes fouling of seawater, increases

332 residence time of live prey all with little or no mechanical turbulence. The diffusion cylinder

333 method can be a critical tool for laboratory use in the cultivation of a variety of pelagic gelata

334 species and thus contribute to a range of research in these fragile organisms on population

335 genetics, zooplankton plastivory, behavior and ecology.

337 Acknowledgements

339 We thank Dr. W.E. Browne for his enthusiastic collaboration, comments on draft manuscript and

340 support of ctenophore culturing here at the aquarium. The DEEP-C project for creating a joint

341 ctenophore project that introduced us to Dr. Browne and other ctenophore researchers. Shannon

342 Johnson for her comments on the draft manuscript. John Negrey for instrument calibration and 
343 support. John Hoech, Paul Clarkson, Marcus Zevalkink, Sarah Halbrend, Evan Firl, the Drifters

344 gallery staff and volunteers that contributed their time and resources to this project.

345

346 Funding

347

348 Funding was provided by the Monterey Bay Aquarium Foundation.

349

350 References

351

352 Dunn CW, Hejnol A, Matus DQ, Pang K, Browne WE, Smith SA, Seaver E, Rouse GW, Obst

353 M, Edgecombe GD, Sørensen MV, Haddock SHD, Schmidt-Rhaesa A, Okusu A, Kristensen

354 RM, Wheeler WC, Martindale MQ, Giribet G. 2008. Broad phylogenomic sampling improves

355 resolution of the animal tree of life. Nature 452:745-749. DOI: 10.1038/nature06614.

356

357 Greve W. 1968. The planktonkreisel, a new device for culturing zooplankton. Marine Biology

358 1:201-203. DOI: 10.1007/BF00347112.

359

360 Greve W. 1970. Cultivation experiments on North Sea ctenophores. Helgoländer

361 Wissenschaftliche Meeresuntersuchungen 20:304-317. DOI: 10.1007/BF01609908.

362

363 Greve W. 1975. The "Meteor Planktonküvette": A device for the maintenance of

364 macrozooplankton aboard ships. Aquaculture 6:77-82. DOI: 10.1016/0044-8486(75)90090-3. 
366 Gyllenberg G, Greve W. 1979. Studies on oxygen uptake in ctenophores. Annales Zoologici

367 Fennici 16:44-49.

368

369 Hamner WM. 1990. Design developments in the planktonkreisel, a plankton aquarium for ships

370 at sea. Journal of Plankton Research 12:397-402. DOI: 10.1093/plankt/12.2.397.

371

372 Hirota J. 1974. Quantitative natural history of Pleurobrachia bachei in La Jolla Bight. Fish. Bull $37372: 295-335$.

374

375 Howes EL, Bednaršek N, Büdenbender J, Comeau S, Doubleday A, Gallager SM, Hopcroft RR,

376 Lischka S, Maas AE, Bijma J, Gattuso J-P. 2014. Sink and swim: a status review of thecosome 377 pteropod culture techniques. Journal of Plankton Research 36:299-315. DOI:

378 10.1093/plankt/fbu002.

379

380 Jaspers C, Marty L, Kiørboe T. 2018. Selection for life-history traits to maximize population

381 growth in an invasive marine species. Global Change Biology 24:1164-1174. DOI:

$38210.1111 / \mathrm{gcb} .13955$.

383

384 Jaspers C, Møller LF, Kiørboe T. 2015. Reproduction rates under variable food conditions and 385 starvation in Mnemiopsis leidyi : significance for the invasion success of a ctenophore. Journal 386 of Plankton Research 37:1011-1018. DOI: 10.1093/plankt/fbv017. 387 
388 Kassambara A. 2019. 'ggplot2' Based Publication Ready Plots. Available at

389 https://rpkgs.datanovia.com/ggpubr/ (accessed on 4 October 2019)

390

391 Martí-Solans J, Ferrández-Roldán A, Godoy-Marín H, Badia-Ramentol J, Torres-Aguila NP,

392 Rodríguez-Marí A, Bouquet JM, Chourrout D, Thompson EM, Albalat R, Cañestro C. 2015.

393 Oikopleura dioica culturing made easy: A Low-Cost facility for an emerging animal model in

394 EvoDevo: oikopleura low cost facility. genesis 53:183-193. DOI: 10.1002/dvg.22800.

395

396 Pang K, Martindale MQ. 2008. Mnemiopsis leidyi Spawning and Embryo Collection. Cold

397 Spring Harbor Protocols 2008:pdb.prot5085-pdb.prot5085. DOI: 10.1101/pdb.prot5085.

398

399 Presnell JS, Browne WE. 2019. Krüppel-like factor gene function in the ctenophore Mnemiopsis

400 suggests an ancient role in promoting cell proliferation in metazoan stem cell niches.

401 Developmental Biology. DOI: 10.1101/527002.

402

403 Presnell JS, Bubel M, Knowles T, Patry W, Browne WE. 2019. Multigenerational laboratory

404 culture of pelagic ctenophores with special focus on the invasive coastal lobate Mnemiopsis

405 leidyi. Nature Protocols, in revision.

406

407 R Core Team. 2017. R: A language and environment for statistical computing. R Foundation for 408 Statistical Computing, Vienna, Austria. Available at https://www.R-project.org/ (Accessed on 4 409 October 2019).

410 
411 Raskoff KA, Sommer FA, Hamner WM, Cross KM. 2003. Collection and Culture Techniques

412 for Gelatinous Zooplankton. The Biological Bulletin 204:68-80. DOI: 10.2307/1543497.

413

414 Schneider CA, Rasband WS, Eliceiri KW. 2012. NIH Image to ImageJ: 25 years of image

415 analysis. Nature Methods 9:671-675. DOI: 10.1038/nmeth.2089.

416

417 Sprouffske K, Wagner A. 2016. Growthcurver: an R package for obtaining interpretable metrics

418 from microbial growth curves. BMC bioinformatics. Available at

419 https://github.com/sprouffske/growthcurver (Accessed on 4 October 2019).

420

421 Wickham H. 2016. ggplot2: Elegant Graphics for Data Analysis. Springer-Verlag New York.

422 Available at http://ggplot2.tidyverse.org (Accessed on 4 October 2019).

423

424 Wickham H, François R, Henry L, Müller K. 2018. dplyr: A Grammar of Data Manipulation. R 425 package version 0.7 .6 . https://CRAN.R-project.org/package=dplyr 


\section{Figure 1}

Experimental setup using three diffusion tubes and 3D rendering of diffusion tubes.

(A) Three sets of diffusion tubes on a wet table. (B) 3D rendering of both tubes with supply tubing at top right. (C) outer tube has been removed to reveal the inner tube resting on a riser made with rigid plastic mesh.

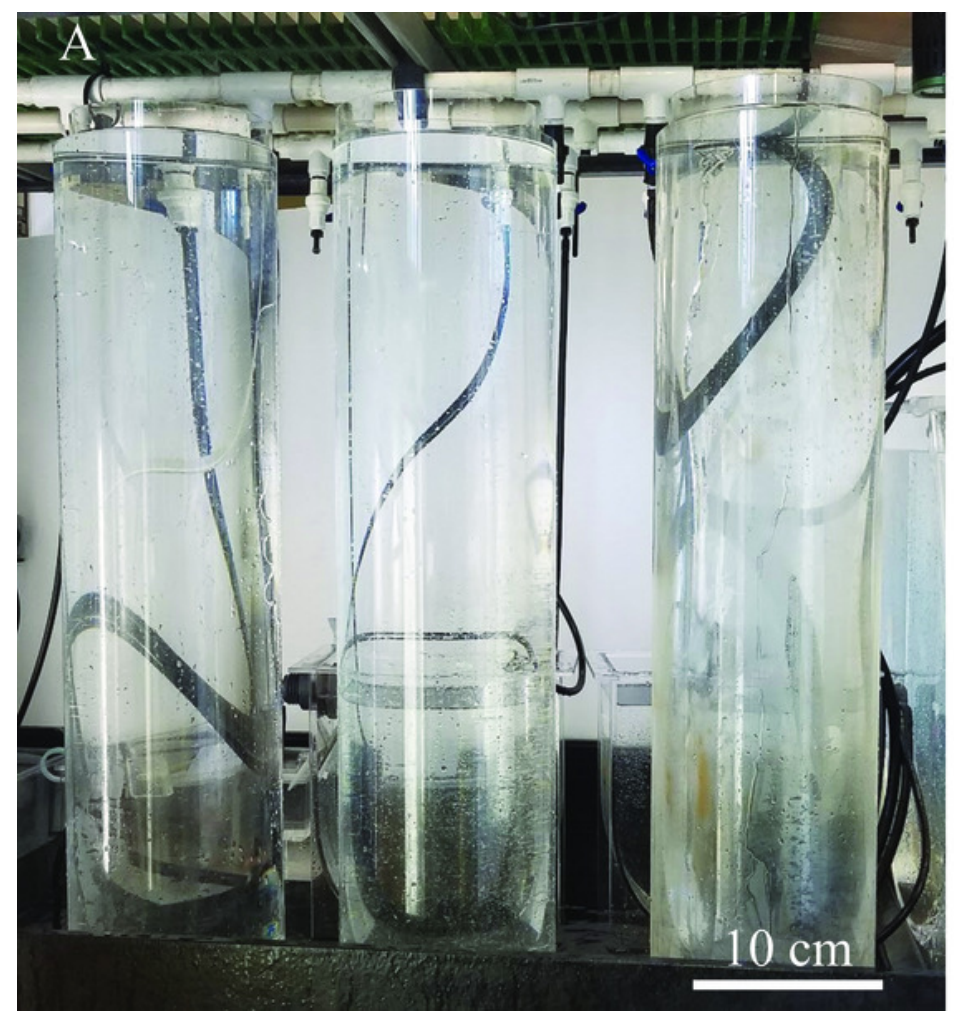

B

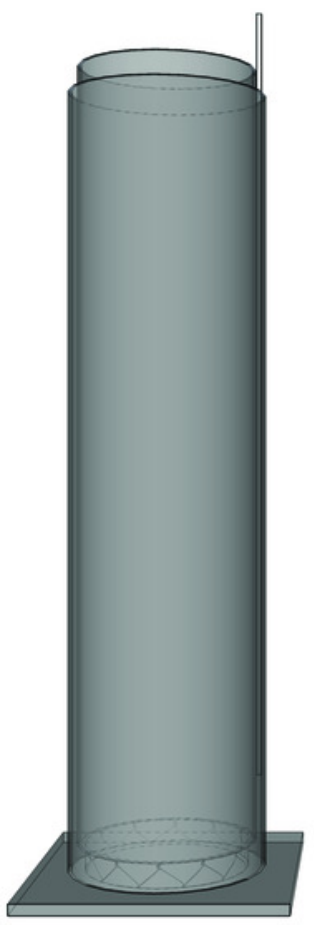

C

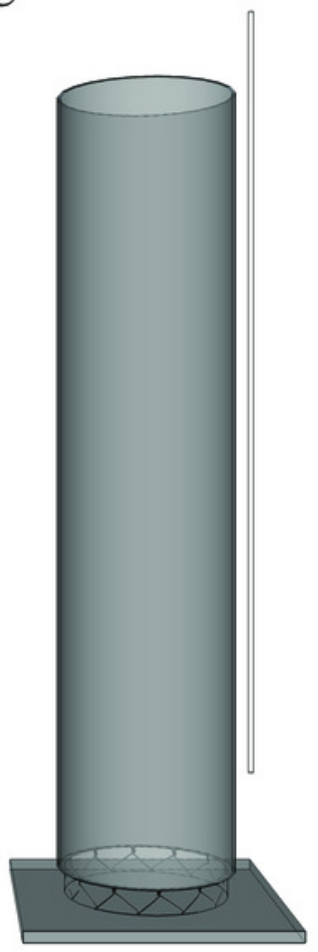


Figure 2

Assembly instructions for diffusion tubes.

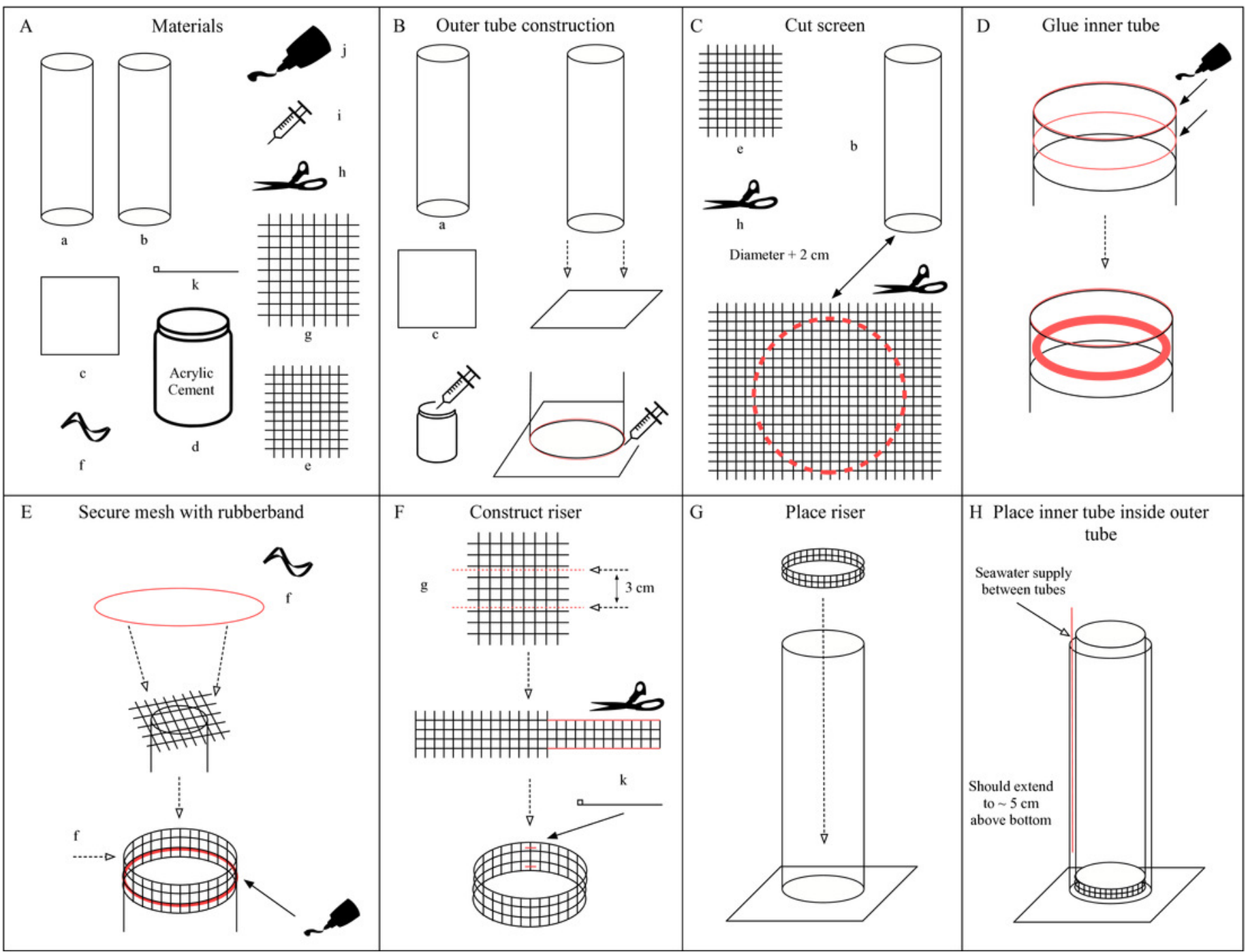




\section{Figure 3}

Spawning ctenophores in the diffusion tubes.

(A) Hormiphora californensis spawning in the diffusion tube with a bright LED light. (B)

Pleurobrachia bachei releasing sperm from the meridional canals beneath the ctene rows.

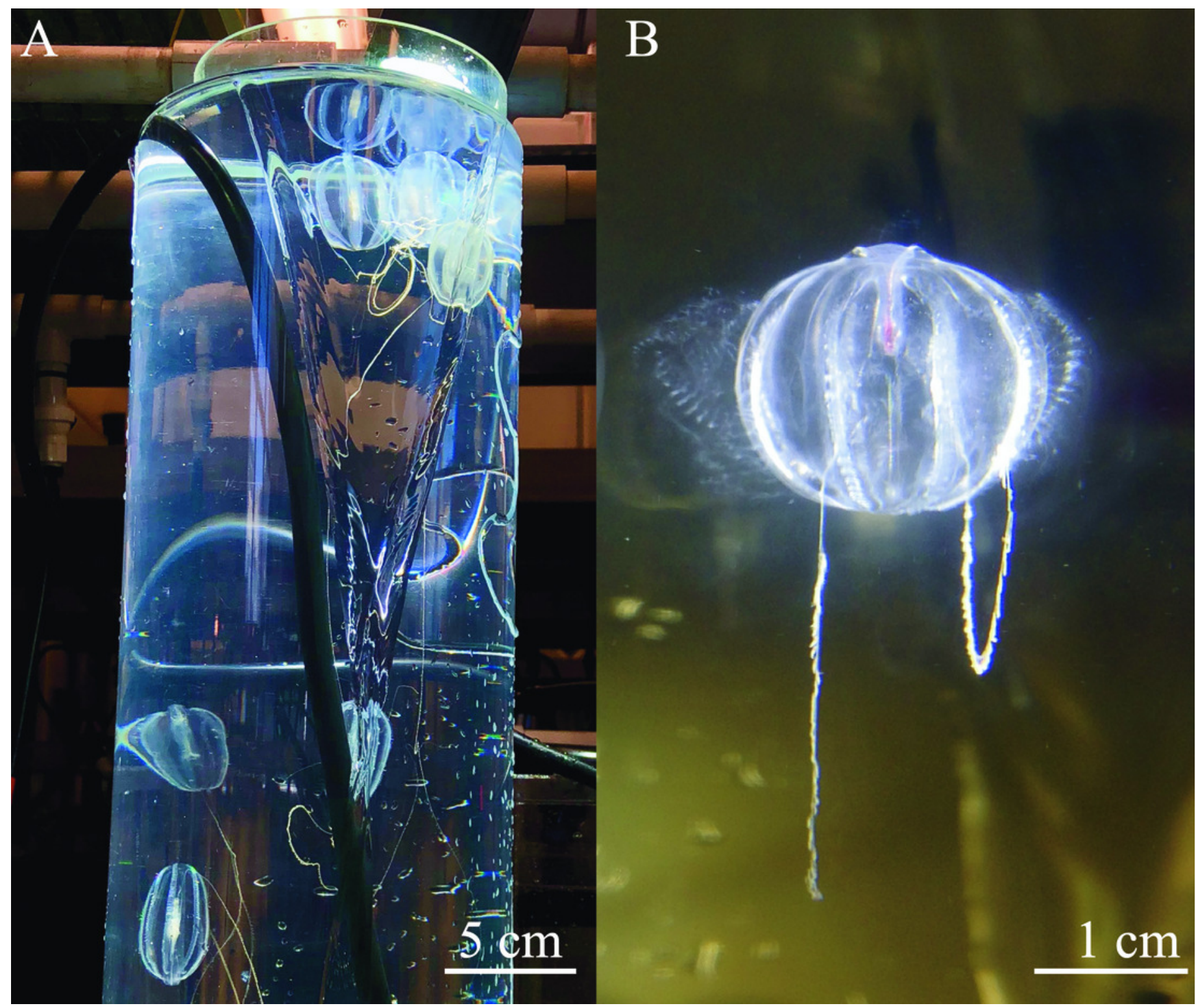


Figure 4

Depth profiles of each treatment for temperature, $\mathrm{pH}$ and dissolved oxygen.

Line graph showing the means (points) of the two measurements taken on opposing sides of the inner tube. (A - C) Temperature by treatment. (D - F) pH by treatment. ( $G$ - I) Dissolved oxygen by treatment. 
Temperature $\left({ }^{\circ} \mathrm{C}\right)$
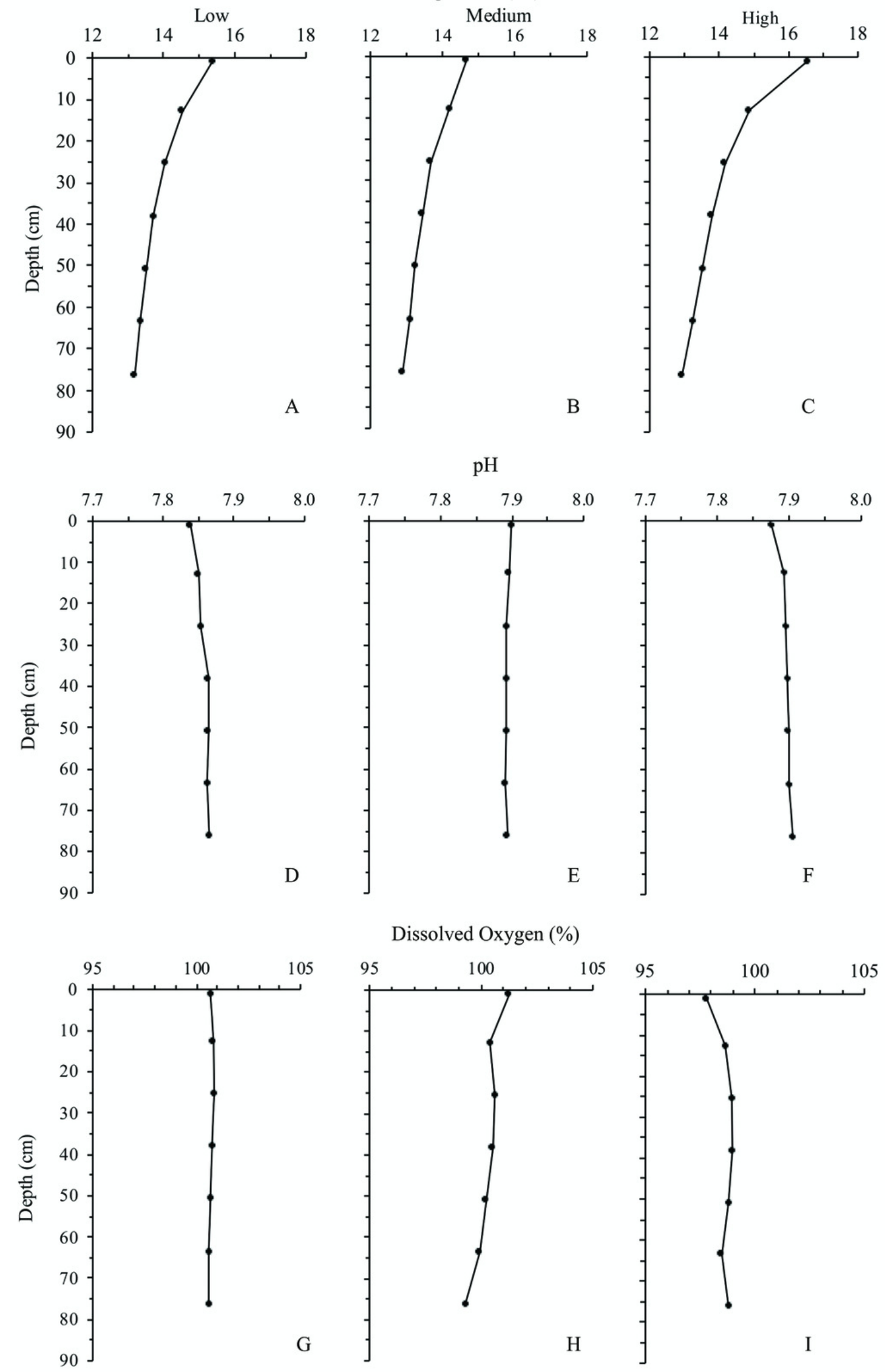
Figure 5

Total length measured over 35 days.

Mean lengths (points) of $H$. californensis with SE (whiskers) in low, medium, and high flow treatments.

\section{Treatment $\square$ LOW $\square$ MED $\square$ HIGH}

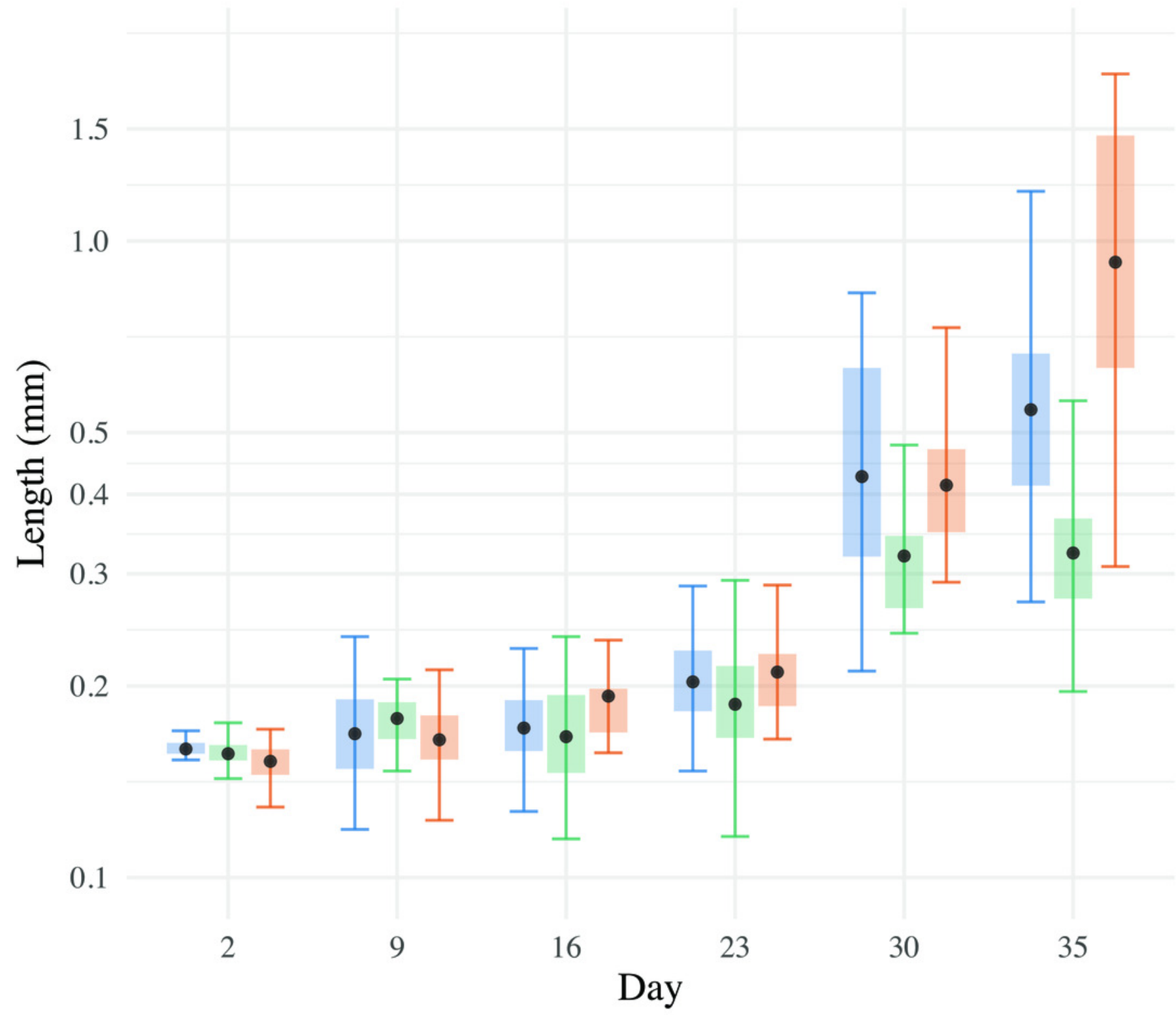




\section{Figure 6}

Pairwise comparison of means for low, medium, and high flow treatments at $35 \mathrm{dph}$.

At $35 \mathrm{dph}$ the high and low flow treatments ended with significantly greater mean

lengths(points) than the medium flow treatment. The high flow treatment had the greatest mean lengths. Whiskers represent $95 \% \mathrm{Cl}$. 


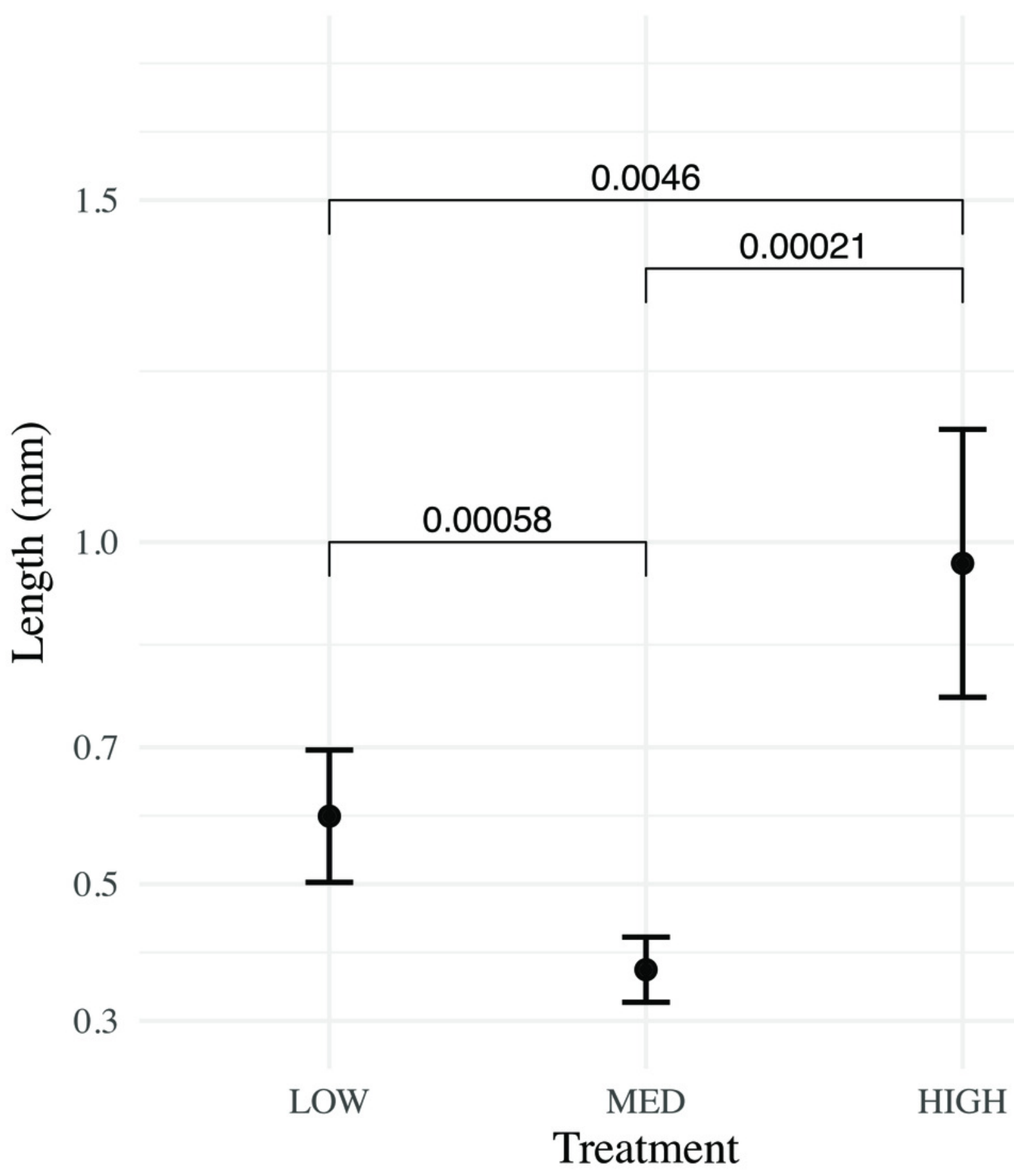




\section{Figure 7}

Taller diffusion tube setup for Leucothea pulchra.

(A) Two meter tall diffusion tubes for rearing Leucothea pulchra sitting inside of a $200 \mathrm{~L}$ reservoir. (B) three large adults were spawned to produce the F1 generation. (C) thermocline visible at the top of tube, $9 \mathrm{dph}$ (D) various srages of F1 larval L. pulchra visible at the surface of the inner tube, $21 \mathrm{dph}$. 


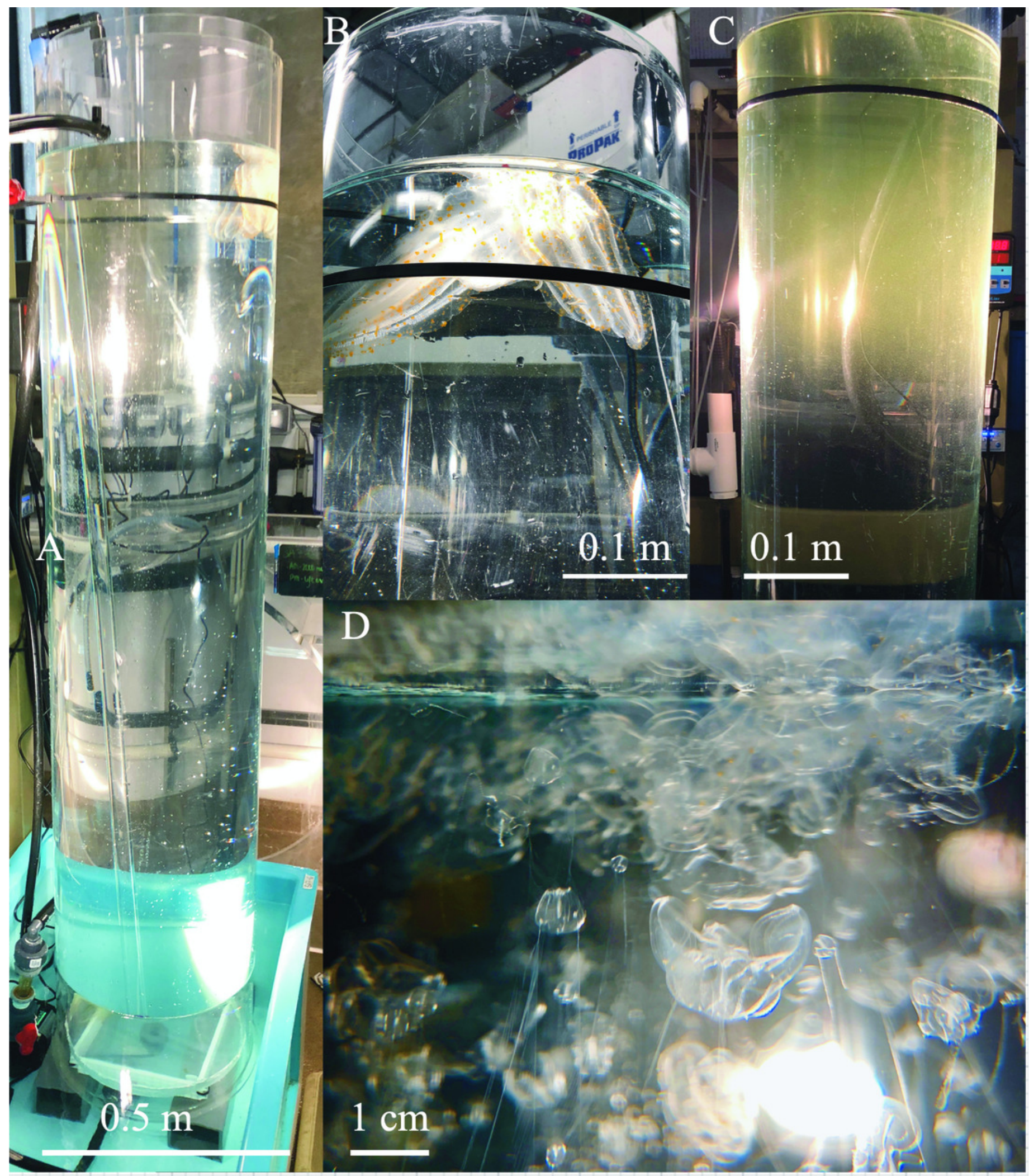


Figure 8

Shorter and wider diffusion tubes for other species of gelata.

(A) $30 \mathrm{~cm}$ wide diffusion tubes used for Bolinopsis infundibulum and Nanomia bijuga culture.

(B) larval N. bijuga at 35 days post settlment visible near the top of the tube (C) mature $B$. infundibulum spawning under bright light, the wider and shorter tube makes it easier to place and remove the adult lobates. 


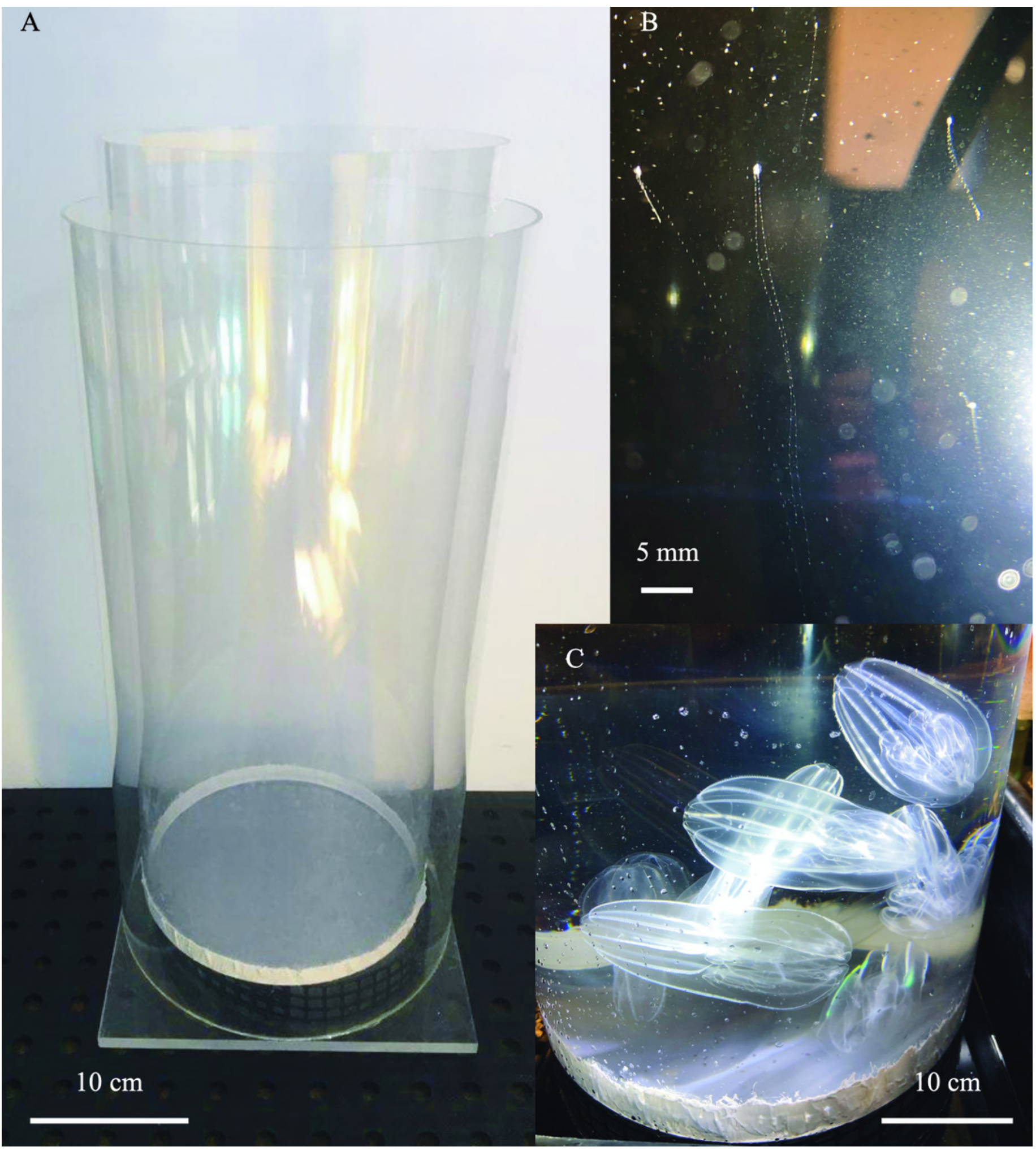




\section{Figure 9}

Detailed feeding protocol for Hormiphora californensis.

Feeds may be skipped when copepods remain in the tube uneaten. When copepods remain in the tube, not consumed, live algae (Isochrysis, Tetraselmis, Rhodomonas etc.) may be added to the next feed as an equal volume to the feed. This helps keep the remaining feed enriched and growing. Feed may be placed in a beaker and slowly dripped into the top of the inner tube using a drip emitter or similar device (DYNALON clamp,part \#670715). Algae cultures are drawn from at different stages of growth, so density varies and should be dosed according to appearance of water color in the upper tube. When algae and feed are added slowly they sit on the thermocline and it is quite apparent when existing copepods have eaten the algae and more should be added. 


\begin{tabular}{|c|c|c|c|c|c|c|c|}
\hline \multicolumn{3}{|c|}{ Pre-hatch } & \multicolumn{5}{|c|}{ Post-hatch } \\
\hline $\begin{array}{l}\text { Development } \\
\text { Stage }\end{array}$ & & & & & & & (1) \\
\hline Time (dph) & 0 & 0 & 1 & 10 & 20 & 30 & 40 \\
\hline Size $(\sim \mathrm{mm})$ & $20-30$ & 0.1 & 0.1 & 0.3 & 1.0 & 4.0 & $>8.0$ \\
\hline Aquaria & $\begin{array}{l}\text { Diffusion } \\
\text { Tube }\end{array}$ & $\begin{array}{l}\text { Diffusion } \\
\text { Tube }\end{array}$ & $\begin{array}{l}\text { Diffusion } \\
\text { Tube }\end{array}$ & $\begin{array}{l}\text { Diffusion } \\
\text { Tube }\end{array}$ & $\begin{array}{l}\text { Diffusion } \\
\text { Tube }\end{array}$ & $\begin{array}{l}\text { Diffusion } \\
\text { Tube }\end{array}$ & $\begin{array}{c}\text { Kreisel or } \\
\text { pseudo-kreisel }\end{array}$ \\
\hline $\begin{array}{c}\text { Feed frequency } \\
\text { (feedings per week) }\end{array}$ & 3 & - & 3 & 3 & $2-3$ & $2-3$ & 2 \\
\hline Copepod nauplii & - & - & $50 \mathrm{~mL}$ & $100 \mathrm{~mL}$ & $200 \mathrm{~mL}$ & $400 \mathrm{~mL}$ & - \\
\hline Adult copepods & - & - & - & $50 \mathrm{~mL}$ & $100 \mathrm{~mL}$ & $200 \mathrm{~mL}$ & $400 \mathrm{~mL}$ \\
\hline $\begin{array}{l}\text { Mysid shrimp } \\
\text { (per cydippid) }\end{array}$ & 2 & - & - & - & - & - & 1 \\
\hline
\end{tabular}


Table $\mathbf{1}$ (on next page)

Growth rates produced using R package: Growthcurver. 
1 Table 1: Growth rates produced using R package: Growthcurver.

2

\begin{tabular}{|c|c|c|c|}
\hline Treatment & $\mathrm{K}$ & $\mathrm{N}$ & $\mathrm{r}$ \\
\hline Low $(1.1 \mathrm{Lpm})$ & 33.759 & 0.013 & 0.635 \\
\hline Med $(2.2 \mathrm{Lpm})$ & - & - & - \\
\hline High $(4.5 \mathrm{Lpm})$ & 33.705 & 0.026 & 0.574 \\
\hline
\end{tabular}


Table 2 (on next page)

Suggested diffusion tube sizes (outer diameters, $\mathrm{mm}$ ) by species. 
1 Table 2: Suggested diffusion tube sizes (outer diameters, $\mathrm{mm}$ ) and flows (Lpm) by species.

\begin{tabular}{|l|c|c|c|c|c|c|}
\hline \multicolumn{1}{|c|}{ Species } & Inner Tube & Thickness & Outer Tube & Thickness & Height & Flow \\
\hline Hormiphora californensis & 203 & 3.175 & 222 & 3.175 & 900 & 5 \\
\hline Pleurobrachia bachei & 127 & 3.175 & 145 & 3.175 & 900 & 5 \\
\hline Bolinopsis infundibulum & 305 & 3.175 & 254 & 3.175 & 600 & 7 \\
\hline Leucothea pulchra & 350 & 3.175 & 381 & 6.35 & 2000 & 10 \\
\hline
\end{tabular}

